\title{
An Analysis on Business Value of Cloud Computing
}

\author{
${ }^{1}$ Tank Pranav, ${ }^{2}$ Sahil Zatakiya \\ ${ }^{1,2}$ Dept. of Computer science \& Engineering B.H. Gardi College of Engineering \& Technology, Rajkot, Gujarat, \\ India
}

\begin{abstract}
The high costs incurred in multiple supplier/Buyer transaction forms the basis for the introduction of a high-value agent service. This type of agent-based model can lower the cost for both buyers and sellers, including monetary, mental and physical expenditure. Cloud computing is not just about cost. It can provide a platform for a business to achieve its ambitions of having an "inclusive" IT system that covers entire supply chain and its customers, enabling and generating business growth more effectively.
\end{abstract}

Keywords: Cloud computing, inclusive, Supply chain

\section{INTRODUCTION}

Cloud is increasing its effect and usage in business along with other emerging technologies. IT sector is perfectly dependent on terms like efficiency, cost and speed. Cloud is comparatively better with this parameters in IT and business sector. The importance of Cloud Computing strategy is increasing on a daily basis, as it moves from an interesting to a "must have" technology for a lot of companies.

According to Gartner, $76 \%$ of 106 conference attendees answered yes when asked whether their enterprise will be pursuing cloud strategy by 2012 [2]. There are many advantages and opportunities to be gained from the Cloud Computing model today and in future that are applicable across the corporate IT and business landscape.

\section{DEFINE CLOUD COMPUTING}

"Cloud Computing is a model for enabling convenient, on-demand network access to a shared pool of configurable computing resources that can be rapidly provisioned and released with minimal management effort or service provider interaction”.

Cloud Computing is internet base computing technology, where resources, software and even information are shared via network. It works like electricity grid, but there is a little difference between grid and cloud[4]. Cloud Computing services use the internet as transmission medium and transform information technology resources into service to end-users including software services, computing platform services, development platform services and base information leasing [1].

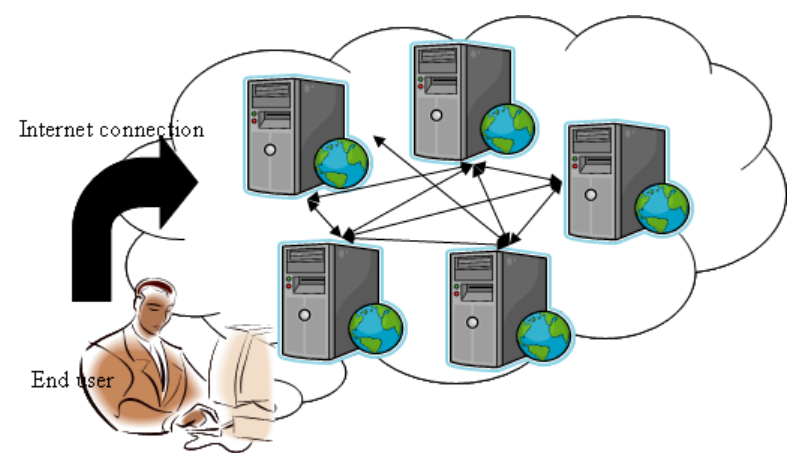

Figurel. Cloud computing concept map

\section{KEY CONCEPTS OF CLOUD COMPUTING}

1. On-Demand Self Service - Customer can provision computing capabilities on-demand basis. Capabilities can be provided automatically without human interactions.

2. Broad network access - Capabilities are available over the network. They can be accessed through standard mechanism with different client platforms.

3. Resource pooling - Service Providers pool capabilities to serve multiple consumers using multi-tenant model.

4. Rapid Elasticity - Capabilities can be rapidly scale in and out at any given time. The Supply of capabilities from user perspective is infinite 
5. Measured Service - Appropriate metering system is employed and customer's usage can be measured, monitored, and reported.

\section{CLOUD COMPUTING SERVICE MODELS}

The three Cloud Computing service models are the following:

1. Cloud Infrastructure as a Service (IAAS) - Provides raw compute, memory and storage transfer capabilities for customer solutions. The target groups for IAAS are infrastructure providers and administrators. An example of this type of service model is Amazon Web Services (aws.amazon.com).

2. Cloud Platform as a Service (PAAS) - Provides development environment for deploying new application onto the cloud. Platforms are offered as application/solution stacks with programming languages and tools. The target groups of PAAS are internet application developer. Examples of this type of service model are Google App Engine (code.google.com/appengine) and Force.com (force.com) application development platforms.

3. Cloud Software as a Service (SAAS) - Provides use of working application running on the provider's cloud infrastructure. Applications are accessed through thin client interface such as web browser. Customer does not undertake control of underlying infrastructure. The target groups of SAAS are application and IT users. Examples of this type of service are Google docs (docs.google.com) and Salesforce customer relationship model (CRM) software (salesforce.com).

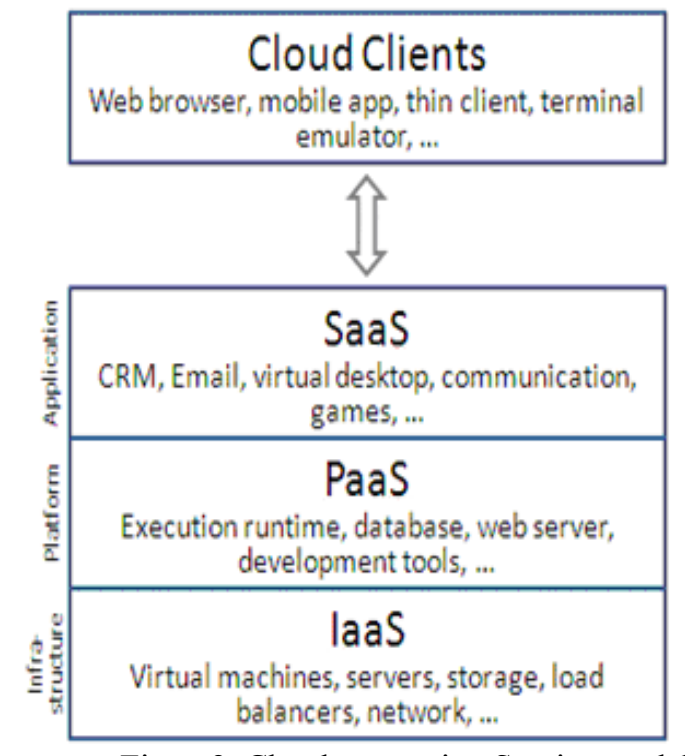

Figure2. Cloud computing Service models

The three-layer architecture or cloud stack is referred as SPI model, where SPI refers to SAAS, PAAS and IAAS respectively. The line between low-level infrastructures to higher-level platform is not crisp and that is why they should be considered together rather than separate entities.

\section{CLOUD COMPUTING DEPLOYMENT MODELS}

Cloud Computing can be set up according to different deployment models such as "Cloud mode" and "Service boundary". There are mainly four different types of Cloud Computing deployment models.

1. Public Cloud - The traditional mainstream sense of cloud computing. The cloud is made available to general public or a large industry group and is owned by an organization providing cloud service. Resources are available from the off-side third party provider who shares resources.

2. Private Cloud - The cloud is operated exclusively for an organization. It is managed by third party or by organization on premise or off premise.

3. Hybrid Cloud - The cloud infrastructure is a composition of two or more clouds that remain unique entities but are bound together by standardized technology that enables data and application portability. It consists of multiple internal or external providers.

4. Community Cloud - The cloud infrastructure is shared by several organizations. It is managed by organization or third party and can exists on or off premise. 


\section{BUSINESS MODELS AND CLOUD}

Cloud is enabler of new approach to IT such as the increasing number of "as a Service" solutions. Few benefits of cloud model are for business infrastructure services,application packages, business logic and data storage. Basic advantage of Cloud is its flexibility for Cloud vendors[5]. Each Cloud model has its own advantage at each layer for Business model.

Now with the revolution, there is also change in business also. Business models have adopted evolution technology as there comfort zone by making Service Oriented Architecture (SOA) and Service Level Agreement (SLA) in developing and deploying model. It also offers a valuable model for software developing and testing. With this new environment business model is also provided with scalability dynamically just as Pay-as-per use mode. The Cloud Computing benefits can be viewed in business as shown in figure 3 [1].

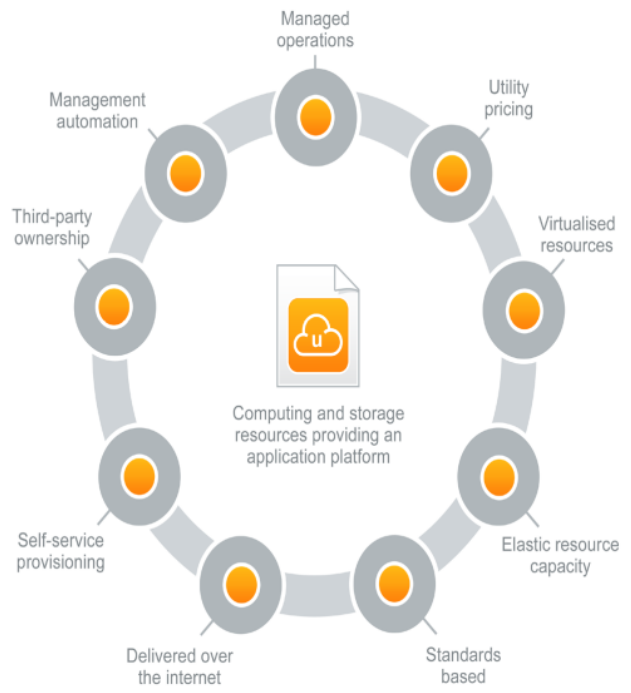

Figure3. Cloud computing Benefits in business model

\section{A. Cloud Computing Business Model}

- Basically providing cloud computing environment service is via hardware and architecture phase. The hardware in a modern environment performs basic function. Operating system is basic block for infrastructure and text processing software.

- Infrastructure of cloud service consists of three different level: infrastructure, platform and application software. The platform level is used in CPU and other hardware resources to execute and access storage media. A single user or different Service providers can build this hierarchy of levels for their business mode.

- The hierarchy of this model presents Platform as a service as Value-added infrastructure service. A special interface is required from user to built infrastructure and while computing platform.

\section{B. User data Privacy}

As we have the facility to lease the service as per need, it helps in saving the cost of software infrastructure. This also helps in storing company's data on service provider's equipment that maybe improperly disclosed. This service can be advantageous to both single service provider as well as to different providers.

The common method to provide data privacy to and protection is by enabling firewall to ensure that decrypted keys that is associated with encrypted data is not disclosed to outside viewers. If both decryption and encryption is associated with the same user then there increases the risk of unauthorized disclosure of user data.

\section{Need Of SOA and SLA}

- System collaboration needs different methods to get supported at business environment, system based on Universal Description Discovery and Integration (UDDI), Web Service Description Language (WSDL) and Simple Object Access Protocol (SOAP) to use web service to transfer Extended Markup Language (XML) form of data.

- Customer Relationship Management (CRM) software is similar type of Service that initiates Data Storage Program, requesting Encryption/Decryption Service System.

- The technique implemented can be used as varying way in enterprise for various needs. SLA provides Agreement to work, it is just like certificating for what one do. This technique provides authentication to data. 
- SOA as mentioned above supports SOA protocol and format the data in XML type which is platform independent thus reduces the time and cost which are the base important factors in business model.

Thus this both techniques over factors above the efficiency and advantage of clouds [5].

\section{THE LONG TAILS IN CLOUD}

One of the fundamental technologies on which Cloud Computing is working today is Virtualization. This technology is used to create large testing environment and to use real world data without disrupting the business.

Uniface customers need to deliver and adapt to environment quickly. Uniface's powerful integration framework and model-driven approach helps to underlying the complexity of new technologies and architecture built and allows much focused functionality and alignment to business goals and needs.

\section{A. Challenges of Cloud}

The model of Cloud always have challenges mainly in architecture,lifecycle management and governing. Many of the challenges will lead to new architectures and delivering target to multiple environment at low cost and complextiy.

Like all other technologies there are few associated cost and management that one might not have considered. So Cloud Computing should be started with practice in business atmosphere with clear goals and targets[4]. Clients will find it familiar and much ease with tutorials and will help them to make a proper and effective patterns of the infrastructure and development.

\section{B. Lack of Tools}

Basically while preparing cloud fit for enterprise many things are to be taken care of and specially when model is SOA driven, as it leverages its strength in developing applications for distributed computing and its support for SOA and Web Services Infrastructure. So with this ability to leverage investments and skills there lack in number of more high profile tools that may clearly help customers to build for cloud.

\section{CONCLUSION AND FUTURE SCOPE}

To access service of cloud user needs to have internet access and so it becomes a mandatory need which at times gets over costed. As service is leased so it needs to be revised when it expires and provider's need to have high-privileged leased service to provide their users with much of benefits.

The future of cloud lies in its ability to withstand with different parameters like infrastructure,platform and software. Cloud users are highly dependend and trusted to the Encryption and Decryption technique provided with SOA and SLA models. So this emphasize users to get activated with this type of services in enterprise as well.

\section{References}

[1] Jing-Jang Hwang and Hung-Kai Chuang, Yi-Chang Hsu and Chien-Hsing Wu, "A Business Model for Cloud Computing based on a separate Encryption and Decryption Service".

[2] A. Weiss, "Computing in the clouds", networker, vol 11, no. 4, pp. 16-25, December 2007.

[3] C.Weinhardt, A.Anandasivam, B.Blau, N.Borissov, "Cloud Computing - a classification, business models and research directions", Business \& Information System Engineering (BISE), vol 1, no. 5

[4] Zhu Bo and Cheng Rentian, "Business model innovations in cloud computing", January 2009.

[5] Jaakko Jaatma, "Financial Aspects Of Cloud Computing Business Models", Aalto School of Economics, January 2010 